\title{
A NOTE ON EXTREME POINTS OF SUBORDINATION CLASSES
}

\author{
D. J. HALLENBECK
}

(Communicated by Irwin Kra)

\begin{abstract}
Let $s(F)$ denote the set of functions subordinate to a univalent function $F$ in $\Delta$ in the unit disc. Let $B$ denote the set of functions $\phi(z)$ analytic in $\Delta$ satisfying $|\phi(x)|<1$ and $\phi(0)=0$. Let $D=F(\Delta)$ and $\lambda(w, \partial D)$ denote the distance between $w$ and $\partial D$ (boundary of $D$ ). We prove that if $\phi$ is an extreme point of $B$ then $\int_{0}^{2 \pi} \log \lambda\left(F\left(\phi\left(e^{i t}\right)\right), \partial D\right) d t=$ $-\infty$. As a corollary we prove that if $F \circ \phi$ is an extreme point of $s(F)$ then $\int_{0}^{2 \pi} \log \lambda\left(F\left(\phi\left(e^{i t}\right)\right), \partial D\right) d t=-\infty$
\end{abstract}

Introduction. Let $\Delta=\{z:|z|<1\}$ and let $\mathbf{A}$ denote the set of functions analytic in $\Delta$. Let $B$ denote the subset of $\mathbf{A}$ consisting of all functions $\phi$ that satisfy the conditions $|\phi(z)|<1, \phi(0)=0$. Let $E B$ denote the extreme points of $B$. Let $S$ denote the subset of $\mathbf{A}$ consisting of univalent functions $f$ so that $f(z)=z+\cdots$ in $\Delta$.

Let $F$ be in $\mathbf{A}$ and be univalent in $\Delta$. Let $s(F)$ denote the subset of $\mathbf{A}$ consisting of functions $f$ that are subordinate to $F$ in $\Delta$. This means that $f \in \mathbf{A}, f(0)=F(0)$, and $f(\Delta) \subset F(\Delta)$. These conditions are equivalent to the existence of $\phi \in B$ so that $f=F \circ \phi$. Note that $s(F)=\{F \circ \phi: \phi \in B\}$.

Let $D$ denote $F(\Delta)$. It is known that $F \in H^{p}$ for all $p<1 / 2[4$, p. 50] and so if $f=F \circ \phi$ for $\phi \in B$ then $f \in H^{p}$ for all $p<1 / 2$ [4, pp. 10-11]. It follows that $\lim _{r \rightarrow 1} f\left(r e^{i t}\right)=f\left(e^{i t}\right)$ exists almost everywhere. In [7] it was proved that $f\left(e^{i t}\right)=F\left(\phi\left(e^{i t}\right)\right)$ for almost all $\theta$. We let $E s(F)$ denote the set of extreme points of $s(F)$. In [1] it was proved that if $F^{\prime}$ is in the Nevanlinna class and $\phi \in E B$ then $\int_{0}^{2 \pi} \log \lambda\left(F\left(\phi\left(e^{i t}\right)\right), \partial D\right) d t=-\infty$. It was conjectured in [1] that the integral was $-\infty$ for any univalent function $F$ when $\phi \in E B$. (Note that this is trivially true if $\left|\phi\left(e^{i t}\right)\right|=1$ on a set of positive measure since $F$ is univalent.) A weaker conjecture is that if $F \circ \phi \in E s(F)$ and $F$ is univalent then $\int_{0}^{2 \pi} \log \lambda\left(F\left(\phi\left(e^{i t}\right)\right), \partial D\right) d t=-\infty$. In a recent paper [2] it was proved that if $F$ is univalent and $\phi \in E B$ then

$$
\int_{0}^{2 \pi} \log \lambda\left(F\left(\phi\left(e^{i t}\right) e^{i \theta}\right), \partial D\right) d t=-\infty
$$

for almost all $\theta$. The analogous form of the weaker conjecture formulated above was also proved in [2].

In this paper we prove both conjectures.

Received by the editors June 12, 1987.

1980 Mathematics Subject Classification (1985 Revision). Primary 30C80; Secondary 30C55.

Key words and phrases. Extreme point, subordination, univalent function. 


\section{Functions subordinate to a univalent function.}

THEOREM 1. If $F$ is a bounded univalent function analytic in $\Delta, \phi \in B$ and $\left|\phi\left(e^{i t}\right)\right|<1$ for almost all $t$, then

$$
\int_{0}^{2 \pi}\left(1-\left|\phi\left(e^{i t}\right)\right|^{2}\right)^{1 / 2}\left|F^{\prime}\left(\phi\left(e^{i t}\right)\right)\right| d t<+\infty
$$

ProOF. Let $g(z)=\int_{0}^{z}\left(F^{\prime}(\tau)\right)^{2} d \tau$ where $z=r e^{i \theta}$ and $\tau=p e^{i \theta}(0 \leq p \leq r)$. Then $g(z)=\int_{0}^{r}\left(F^{\prime}\left(p e^{i \theta}\right)\right)^{2} i e^{i \theta} d p$. Since $F$ is a bounded univalent function, $F(\Delta)$ has finite area. Hence,

$$
\int_{0}^{2 \pi}\left|g\left(r e^{i \theta}\right)\right| d \theta \leq \int_{0}^{2 \pi}\left(\int_{0}^{r}\left|F^{\prime}\left(p e^{i \theta}\right)\right|^{2} d p\right) d \theta<+\infty
$$

It follows from (2) that $g \in H^{1}$ and so $[4$, p. 2] we have by analytic completion

$$
g(z)=\frac{1}{2 \pi} \int_{0}^{2 \pi} \frac{e^{i t}+z}{e^{i t}-z} d \mu(t)+i \beta
$$

where $\mu(t)$ is a function of bounded variation on $[0,2 \pi]$ and $\beta$ is a real constant. Since $g^{\prime}(z)=\left(F^{\prime}(z)\right)^{2}$ it follows from (3) that

$$
\left(F^{\prime}(z)\right)^{2}=\frac{1}{\pi} \int_{0}^{2 \pi} \frac{e^{i t}}{\left(e^{i t}-z\right)^{2}} d \mu(t) .
$$

We deduce from (4) that

$$
\left(1-|z|^{2}\right)\left|F^{\prime}(z)\right|^{2} \leq \frac{1}{\pi} \int_{0}^{2 \pi} \frac{1-r^{2}}{1-2 r \cos (\theta-t)+r^{2}}|d \mu(t)| .
$$

Denote the right-hand side of $(5)$ by $u(z)$. Since

$$
w(z)=\frac{1}{\pi} \int_{0}^{2 \pi} \frac{e^{i t}+z}{e^{i t}-z}|d \mu(t)|
$$

is analytic in $\Delta$ and $u(z)=\operatorname{Re} w(z)$ we conclude that $u(z)$ is harmonic in $\Delta$.

The function $\left(1-\left|\phi\left(e^{i t}\right)\right|^{2}\right)\left|F^{\prime}\left(\phi\left(e^{i t}\right)\right)\right|^{2}$ is positive and measurable since $\left|\phi\left(e^{i t}\right)\right|<$ 1 for almost all $t$ and $\left(1-\left|\phi\left(r e^{i t}\right)\right|^{2}\right)\left|F^{\prime}\left(\phi\left(r e^{i t}\right)\right)\right|^{2}$ is continuous. It follows from Fatou's lemma that

(6) $\int_{0}^{2 \pi}\left(1-\left|\phi\left(e^{i t}\right)\right|^{2}\right)\left|F^{\prime}\left(\phi\left(e^{i t}\right)\right)\right|^{2} d t \leq \lim _{r \rightarrow 1} \int_{0}^{2 \pi}\left(1-\left|\phi\left(r e^{i t}\right)\right|^{2}\right)\left|F^{\prime}\left(\phi\left(r e^{i t}\right)\right)\right|^{2} d t$.

We conclude from (5) and (6) that

$$
\int_{0}^{2 \pi}\left(1-\left|\phi\left(e^{i t}\right)\right|^{2}\right)\left|F^{\prime}\left(\phi\left(e^{i t}\right)\right)\right|^{2} d t \leq \lim _{r \rightarrow 1} \int_{0}^{2 \pi} u\left(\phi\left(r e^{i t}\right)\right) d t .
$$

Since $u(\phi(z))$ is harmonic in $\Delta$ and $\phi(0)=0$, the right-hand side of (7) is equal to $2 \pi u(0)$. Hence,

$$
\int_{0}^{2 \pi}\left(1-\left|\phi\left(e^{i t}\right)\right|^{2}\right)\left|F^{\prime}\left(\phi\left(e^{i t}\right)\right)\right|^{2} d t<+\infty
$$

We note that (1) follows from (8) by an application of the Cauchy-Schwarz inequality. This completes the proof.

We next prove our main theorem. 
THEOREM 2. If $F$ is a univalenvt function analytic in $\Delta, \phi \in E B$, then

$$
\int_{0}^{2 \pi} \log \lambda\left(F\left(\phi\left(e^{i t}\right)\right), \partial D\right) d t=-\infty
$$

PROOF. We first note that by arguments given in detail in [2] it is sufficient to consider the case that $F$ is bounded. Since (9) is easily seen to hold with $\left|\phi\left(e^{i t}\right)\right|=1$ on a set of positive measure we only consider the case $\left|\phi\left(e^{i t}\right)\right|<1$ for almost all $t$. By Theorem (1) we know that (1) holds. It is easy to deduce from (1) that

$$
\int_{0}^{2 \pi} \log \left[\left(1-\left|\phi\left(e^{i t}\right)\right|^{2}\right)^{1 / 2}\left|F^{\prime}\left(\phi\left(e^{i t}\right)\right)\right|\right] d t<+\infty .
$$

Since $F$ is univalent, it follows from [6, p. 22] that

$$
\lambda\left(F\left(\phi\left(e^{i t}\right)\right), \partial D\right) \leq\left(1-\left|\phi\left(e^{i t}\right)\right|^{2}\right)\left|F^{\prime}\left(\phi\left(e^{i t}\right)\right)\right|
$$

for almost all $t$. It follows from (11) that we have

$$
\begin{gathered}
\int_{0}^{2 \pi} \log \lambda\left(F\left(\phi\left(e^{i t}\right)\right), \partial D\right) d t \leq \frac{1}{2} \int_{0}^{2 \pi} \log \left(1-\left|\phi\left(e^{i t}\right)\right|^{2}\right) d t \\
+\int_{0}^{2 \pi} \log \left[\left(1-\left|\phi\left(e^{i t}\right)\right|^{2}\right)^{1 / 2}\left|F^{\prime}\left(\phi\left(e^{i t}\right)\right)\right|\right] d t .
\end{gathered}
$$

Since $\phi \in E B$ we have $\int_{0}^{2 \pi} \log \left(1-\left|\phi\left(e^{i t}\right)\right|^{2}\right) d t=-\infty[4$, p. 125] and so (9) follows from this fact, (10) and (12). This completes the proof.

THEOREM 3. If $F$ is a univalent function analytic in $\Delta, \phi \in B$ and $F \circ \phi \in$ $E s(F)$ then

$$
\int_{0}^{2 \pi} \log \lambda\left(F\left(\phi\left(e^{i t}\right)\right), \partial D\right) d t=-\infty
$$

Proof. This follows from Theorem 2 above and Theorem 1 in [2] where it was proved that if $F \circ \phi \in E s(F)$ then $\phi \in E B$.

REMARK. Condition (13) is seen to be a necessary condition for $F \circ \phi \in E s(F)$. Also, it is known that $\int_{0}^{2 \pi} \log \lambda\left(F\left(\phi\left(e^{i t}\right)\right), \partial D\right) d t=-\infty$ does not in general imply that $F \circ \phi \in E s(F)$. This can be easily seen by considering the case $F(z)=$ $((1+z) /(1-z))^{\alpha}$ for $0<\alpha \leq 1[4$, pp. 131, 133].

THEOREM 4. Suppose $F$ is a univalent function analytic in $\Delta$ and $\phi \in B$. Then

$$
\int_{0}^{2 \pi} \log \lambda\left(F\left(\phi\left(e^{i t}\right)\right), \partial D\right) d t=-\infty
$$

if and only if $\phi \in E B$.

ProOF. If $\phi \in E B$ then (14) follows from Theorem 3. The other implication was proved in [5].

\section{REFERENCES}

1. Y. Abu-Muhanna, On extreme points of subordination families, Proc. Amer. Math. Soc. 87 (1983), 439-443.

2. Y. Abu-Muhanna and D. J. Hallenbeck, Subordination families and extreme points, Trans. Amer. Math. Soc. 308 (1988), 83-89. 
3. L. Brickman, T. H. MacGregor, and D. R. Wilken, Convex hulls of some classical families of univalent functions, Trans. Amer. Math. Soc. 156 (1971), 91-107.

4. P. L. Duren, Theory of $H^{p}$ spaces, Academic Press, New York, 1970.

5. D. J. Hallenbeck and T. H. MacGregor, Linear problems and convexity techniques in geometric function theory, Monographs and Studies in Math., vol. 22, Pitman, 1984.

6. Chr. Rommerenke, Univalent functions, Vandenhoeck and Ruprecht, Göttingen, 1975.

7. J. V. Ryff, Subordinate $H^{p}$ functions, Duke Math. J. 33 (1966), 347-354.

Department of Mathematical Sciences, University of Delaware, Newark, DELAWARE 19716 